\title{
Hodographs of the Gravitational Two-Body System and Discrepancies between Newtonian Laws of Equivalent Kepler Orbits and General Relativity
}

\author{
Caesar P. Viazminsky ${ }^{1,2}$, Piere K. Vizminiska ${ }^{3}$ \\ ${ }^{1}$ Department of Physics, Illinois Institute of Technology, Chicago, USA \\ ${ }^{2}$ Department of Physics, University of Aleppo, Aleppo, Syria \\ ${ }^{3}$ Department of Computer Engineering, University of Detroit Mercy, Detroit, USA \\ Email: kaysarv2@gmail.com
}

Received October 22, 2013; revised November 22, 2013; accepted November 29, 2013

Copyright (C) 2014 Caesar P. Viazminsky, Piere K. Vizminiska. This is an open access article distributed under the Creative Commons Attribution License, which permits unrestricted use, distribution, and reproduction in any medium, provided the original work is properly cited. In accordance of the Creative Commons Attribution License all Copyrights (C) 2014 are reserved for SCIRP and the owner of the intellectual property Caesar P. Viazminsky, Piere K. Vizminiska. All Copyright (C) 2014 are guarded by law and by SCIRP as a guardian.

\section{ABSTRACT}

We show first that an orbit, which is naturally characterized by its eccentricity and semi-latus rectum, can equally be characterized by other sets of parameters, and proceed to determine mass-independent characterizations. The latter is employed to obtain the laws of equivalent orbits, which by definition have the same eccentricity and orbit's parameter [1]. These laws relate the values of the same physical observables on two equivalent orbits to the corresponding total mass; they include the laws of velocity, angular velocity, radial velocity, areal velocity, acceleration, period, energy and angular momentum. Regardless of the share of the two bodies of a fixed total mass, the same relative orbit occurs for the same initial conditions. Moreover, the same orbit can be traced by different total masses but with different relative velocities. The concept of a gravitational field generated by a set of masses is shown to be meaningful only when the center of mass is not changed by the test mass. The associated concept of the "nothing", which is an infinitesimal mass that allows for the property just mentioned to be fulfilled, is introduced and its orbits are determined. The perturbation of the nothing orbits due to its replacement by a finite mass is determined. It is proved that such a replacement can have a qualitative effect resulting in a "phase transition" of an orbit from unbound to bound, and that the nothing's circular orbits cannot be occupied by any material body. The Galileo law of free fall, on which the equivalence principle hinges and which is exact only for "nothing-like" falling objects, is revised to determine the duration of free fall of a body of an arbitrary mass. The wholeness of Newton's laws and the associated concept of force as an interaction are highlighted, and some contradictions between the Newtonian laws of equivalent Kepler's orbits and the general relativistic predictions are discussed. It is demonstrated that Newton's law of gravitation is not an approximation of Einstein field Equations even in the case of a static weak field. However, both theories have a common limit corresponding to the case in which the alien concept of a field can be incorporated in the Newtonian theory. We also show that the relative velocity's hodograph [2-4], the alternative Laplace-Runge-Lenz (LRL) vector derived by Hamilton [4-6], as well as an infinite set of LRL vectors, result all from one vector. The hodograph is a proper circular arc for hyperbolic motion, a circle less a point for parabolic motion, and a full circle for bound motion.

\section{KEYWORDS}

Hodographs; LRL Vector; Laws of Equivalent Orbits; Revised Galileo Law of Free Fall; Equivalence Principle

\section{Introduction}

Beside the main subject discussed in this article, which is the laws of equivalent Kepler orbits and its contradiction with the predictions of general relativity (GR), a separate issue concerning the velocity hodographs is dis- 
cussed in Section 3, and an LRL vector is derived from which the velocity hodograph follows immediately.

The body of the main subject of this article can be summarized by the following:

- We set up first a variety of characterizations of an orbit that are equivalent to its natural one, and generalize it to a mass-independent form.

- Orbits that can be made to coincide through rotations, translations, or reflections are called equivalent. Based on the mass-independent characterization of an orbit we obtain the laws of equivalent relative orbits.

- Neglecting the alteration of the center of mass, the same initial conditions yield the same relative orbit independently of the share of the two bodies of a fixed total mass. Moreover, the same relative orbit with different total mass occurs when the relative velocity is proportional to the square root of the total mass.

- The concept of a gravitational field generated by a set of masses is shown to be meaningful only when the passive test body's mass is negligible. The useful concept of the "nothing" is accordingly introduced and its orbits are determined.

- The perturbation in a nothing's orbits due to its replacement by a finite mass is determined. It is proved that such a replacement can change the nature of motion from unbound to bound. It was also shown that the nothing's circular orbits cannot be occupied by any material body.

- The Galileo law of free fall (GLFF), on which the equivalence principle hinges, and which is accurate only for "nothing-like" falling objects, is revised to state that: the duration of a free fall by a given distance is inversely proportional to the square root of the total mass.

- Some contradictions between the Newtonian laws of equivalent orbits and the predictions of GR are discussed. It is also shown that Newton's law of gravitation is not an approximation of Einstein field Equations even in the case of a static weak field.

Before starting to implement our plan we brief an essential background of the subject.

We refer a system of two particles with masses $m_{1}$ and $m_{2}$ to the inertial frame $S_{c} \equiv C x y z$ with origin $C$ at the center of mass and axes' units $(\boldsymbol{i}, \boldsymbol{j}, \boldsymbol{k})$. If $\boldsymbol{r}_{1}\left(\boldsymbol{r}_{2}\right)$ is the position vector of the first (second) particle in $S_{c}$, then their relative position and velocity are $r=r_{2}-r_{1}$, and $\boldsymbol{v}=\boldsymbol{v}_{2}-\boldsymbol{v}_{1}$ respectively. If the particles interact only through gravitation then the relative acceleration, $\boldsymbol{a}=\boldsymbol{a}_{2}-\boldsymbol{a}_{1}$, of the system is $[1,7]$

$$
\boldsymbol{a}=-\left(G M / r^{2}\right) \boldsymbol{I},
$$

where $G$ is the gravitational constant, $M=m_{1}+m_{2}$ is the total mass of the system, and $\boldsymbol{I}=\boldsymbol{r} / \boldsymbol{r}$ (Figure 1 ). We assume that $m_{1}>m_{2}$. The equation of motion (1.1) shows that the relative motion can be described by quantities that are independent of the distribution of the total mass between the individual particles. Employing polar coordinates $(r, \theta)$ in the plane of motion $[\boldsymbol{i}, \boldsymbol{j}] \equiv C x y$, with $C x$ is the polar axis, the conserved system's mechanical energy $E$ and angular momentum $\boldsymbol{L}=\mu \boldsymbol{h}=\mu h \boldsymbol{k}$ can be expressed by the equations [1]:

$$
\begin{aligned}
& 2 \varepsilon \equiv \frac{2 E}{\mu}=\dot{r}^{2}+\frac{h^{2}}{r^{2}}-\frac{2 G M}{r}, \\
& h=\frac{L}{\mu}=r^{2} \dot{\theta},
\end{aligned}
$$

where $\mu$ is the reduced mass. The quantities $\varepsilon$ and $h$ are called the reduced energy and angular momentum densities respectively [1].

The orbit of the system is found by a well-known method [7-9]:

$$
\boldsymbol{r}=P \boldsymbol{I} /\left[1+e \cos \left(\theta-\theta_{p}\right)\right],
$$

where $\theta_{p}$ is a constant of integration that depends only on the choice of the polar axis, and

$$
\begin{gathered}
P=h^{2} / G M, \\
e=\left(1+2 \varepsilon h^{2} / G^{2} M^{2}\right)^{1 / 2},
\end{gathered}
$$

are the semi-latus rectum and the eccentricity of the orbit. For simplicity we assume that the polar axis is chosen to pass through the perihelion, and hence $\theta_{p}=0$ in (1.3). 


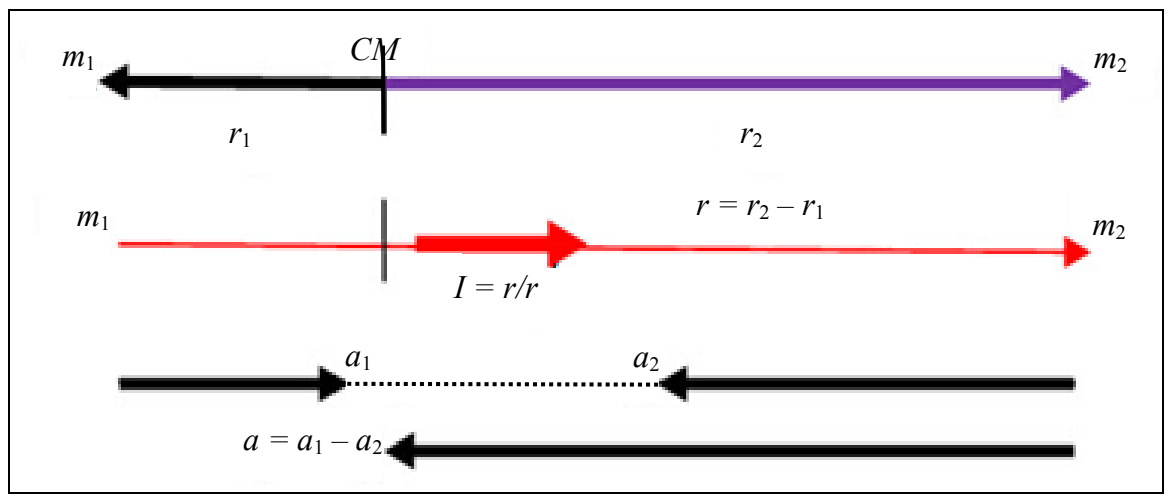

Figure 1. The relative acceleration points against the relative position vector.

The trajectory $r=r(\theta)$ of the system, or the relative orbit, may refer to the trajectory of either particle, say $m_{2}$ in a frame $s_{1}$ co-moving with $m_{1}$, and not rotating relative to the fixed stars; the focus of the relative orbit in this choice is at $m_{1}$. Or instead, the trajectory $r=r(\theta)$ is the relative orbit in the frame $S_{c}$, in which case the measured length is $r(\theta)=r_{1}(\theta)+r_{2}(\theta)$ and $C M$ is the origin of the polar coordinates. The relative orbit in $S_{c}$ in this case is the abstract locus $r=r(\theta)$ with focus at the $C M$, and the orbit is merely the collection of the pairs $\left(r_{1}(\theta)+r_{2}(\theta), \theta\right)$ at various instants of time referred to a polar system with origin at $C M$.

The orbits of the individual particles in $S_{c}$ are [1]

$$
\begin{aligned}
& \boldsymbol{r}_{1}(\theta)=-\boldsymbol{I} \frac{\left(m_{2} / M\right) P}{1+e \cos \theta}, \\
& \boldsymbol{r}_{2}(\theta)=\boldsymbol{I} \frac{\left(m_{1} / M\right) P}{1+e \cos \theta} .
\end{aligned}
$$

\section{Equivalent Characterizations of an Orbit}

We may look on one orbit $\{P, e\}$ as a representative of a class of equivalence of orbits which result from one orbit through rotation, inversion, or translation. The latter fact follows from the homogeneity of the space with respect to our closed two-body system, and hence its isotropy [10].

We present here various characterizations of a class of orbits $\{P, e\} \quad[1]$. At an arbitrary point of the system's trajectory, both components of the relative velocity $\boldsymbol{v}=v_{I} \boldsymbol{I}+v_{J} \boldsymbol{J}$ in the moving system $(\boldsymbol{I}, \boldsymbol{J})$ are non-zero in general. At the perihelion, $r=r_{p}$, the radial velocity vanishes $\left(v_{I}=\dot{r}=0\right)$, and the velocity is purely tangential; it is given by $v_{p}=r_{p} \dot{\theta}=h / r_{p}$. In terms of $\left(r_{p}, v_{p}\right)$ we write (1.4) as follows [1]:

$$
\text { (i) } P=\frac{r_{p}^{2} v_{p}^{2}}{G M}, \text { (ii) } e=\frac{r_{p} v_{p}^{2}}{G M}-1 \text {. }
$$

By (1.4ii) and (2.1ii) we obtain the equivalent inequalities

$$
\text { (i) } 2 \varepsilon h^{2} \geq-G^{2} M^{2} \text {, (ii) } r_{p} v_{p}^{2} \geq G M \text {. }
$$

Assuming that the motion takes place in the positive sense $(\dot{\theta}>0)$ and $M$ is fixed, it is easy to show that the pairs

$$
\{P, e\},\left\{r_{p}, v_{p}\right\},\{h, \varepsilon\},
$$

are in 1-1 correspondence. Changing the variable $v$ to $\vartheta=v / \sqrt{M}$, each of the pairs

$$
\{P, e\},\left\{r_{p}, \vartheta_{p}\right\},\{\bar{h} \equiv h / \sqrt{M}, \bar{\varepsilon} \equiv \varepsilon / M\},
$$


can be expressed conclusively in terms of $\left\{r_{p}, \vartheta_{p}\right\}$ and independently of the total mass $M$. Again, it is easy to prove that the pairs (2.4) are in $1-1$ correspondence. This means that there corresponds to each given value of one pair unique values to other pairs, and therefore, the same orbit $\{P, e\}$ is also realizable by the corresponding value of $\left\{r_{p}, \vartheta_{p}\right\}$ or $\{\bar{h}, \bar{\varepsilon}\}$. Thus the same orbit can be realized for different values of $M$ provided $r_{p}$ is kept fixed and $v_{p}$ is proportional to $\sqrt{M}$. Contrary to the correspondences (2.3) in which $M$ is fixed, $M$ in (2.4) is arbitrary, and thus it determines orbits with the same $\{P, e\}$ and different total masses. The correspondences (2.3) are a particular case of (2.4) in which $M$ becomes fixed.

\section{Hodographs and Laplace-Runge-Lenz Vectors}

We have shown in [1] that, upon knowing the energy and the angular momentum vector, any additional independent constant of motion can only specify the axis of symmetry of the orbit, i.e. the angle $\theta_{p}$ it makes with the polar axis. In particular, the role of the LRL vector under the given data, is confined to determine the orientation of the orbit in the plane of motion.

The LRL vector emerges from the inverse square law (1.1)

$$
\frac{\mathrm{d} v}{\mathrm{~d} t}=-\frac{G M}{r^{2}} \boldsymbol{I}=-\frac{G M}{h} \dot{\theta} \boldsymbol{I}=\frac{G M}{h} \frac{\mathrm{d} \boldsymbol{J}}{\mathrm{d} t},
$$

which shows that the vector field

$$
\boldsymbol{B} \equiv h \boldsymbol{v}-G M \boldsymbol{J}=e G M \boldsymbol{J}_{p}
$$

is constant of motion. The constant value of this vector on an orbit is obtained from its value at the perihelion

$$
\boldsymbol{B}=\left(r_{p} v_{p}^{2}-G M\right) \boldsymbol{J}_{p}=e G M \boldsymbol{J}_{p},
$$

where equation (2.1ii) has been used. It is clear that the vector field $\boldsymbol{B}$ which is defined on an orbit is perpendicular to its axis of symmetry since it always has the value $e G M \boldsymbol{J}_{p}$. Because $\boldsymbol{B}$ makes an angle $\pi / 2+\theta_{p}$ with the polar axis, the orientation of an orbit is determined by the angle $\theta_{p}$ between its axis of symmetry and the polar axis.

The familiar LRL vector [1-6] is obtained by taking the cross product of both sides of (3.2) from right by $\boldsymbol{k}$

$$
\boldsymbol{A}=\boldsymbol{B} \times \boldsymbol{k} \equiv \boldsymbol{v} \times \boldsymbol{h}-G M \boldsymbol{I}=e G M \boldsymbol{I}_{p} .
$$

It is clear that $A=B=e G M, \quad \boldsymbol{A} \times \boldsymbol{B}=A^{2} \boldsymbol{k}, \boldsymbol{A} \cdot \boldsymbol{B}=\boldsymbol{L} \cdot \boldsymbol{A}=\boldsymbol{L} \cdot \boldsymbol{B}=0$. It is to be noted that the alternative LRL vector $\boldsymbol{B}_{\boldsymbol{H}}$ found by Hamilton [4-6]

$$
\boldsymbol{B}_{\boldsymbol{H}}=\boldsymbol{p}-\left(\frac{G m_{1} m_{2}^{2}}{L^{2} r}\right) \boldsymbol{L} \times \boldsymbol{r}
$$

is an approximation of the vector $\mu \boldsymbol{B} / h$. Indeed

$$
\boldsymbol{B}_{H}=m_{2} \boldsymbol{v}_{2}-\left(\frac{G m_{1} m_{2}^{2}}{m_{2}^{2} h^{2}}\right) m_{2} h \boldsymbol{k} \times \boldsymbol{I}=m_{2} \boldsymbol{v}_{2}-\frac{G m_{1} m_{2}}{h} \boldsymbol{J} .
$$

When the mass $m_{1}$ is dominant, $m_{2} \boldsymbol{v}_{2} \approx \mu \boldsymbol{v}$, and hence

$$
\boldsymbol{B}_{H}=\mu \boldsymbol{v}-\frac{G \mu M}{h} \boldsymbol{J}=\frac{\mu \boldsymbol{B}}{h} .
$$

Assume that the angular momentum $\boldsymbol{L}=\mu h \boldsymbol{k}$ of the system is known, and hence the plane of motion is known. Now, each orbit $\left(P, e, \theta_{p}\right)$ determines a vector field $\boldsymbol{B}$ which takes the value $e M G \boldsymbol{J}_{p}$ at all points of the given orbit. We shall show that the converse is true; each vector field $\boldsymbol{B} \equiv h v-G M \boldsymbol{J}=e M G \boldsymbol{J}_{p}$ determines the orbit $\left(P, e, \theta_{p}\right)$.

Theorem: The mapping $\left(P, e, \theta_{p}\right) \leftrightarrow \boldsymbol{B}=e M G \boldsymbol{J}_{p}$ is $1-1$.

Proof: Let $\boldsymbol{I}_{p}=\boldsymbol{I}\left(\theta_{p}\right)$ be the value of $\boldsymbol{I}$ at a point $\left(r_{p}, \theta_{p}\right)$ of an arbitrary relative possible orbit $r=r(\theta)$ of two gravitating bodies. Now: 


$$
\begin{aligned}
\boldsymbol{B} \equiv & h \boldsymbol{v}-G M \boldsymbol{J}=e M G \boldsymbol{J}_{p} \text { on the orbit } r=r(\theta) \\
\leftrightarrow & \boldsymbol{A}=\boldsymbol{v} \times \boldsymbol{h}-G M I=e G M \boldsymbol{I}_{p} \text { on the orbit } r=r(\theta) \\
\leftrightarrow & G M \boldsymbol{r} \cdot\left[\boldsymbol{I}+e \boldsymbol{I}_{p}\right]=\boldsymbol{r} \cdot(\boldsymbol{v} \times \boldsymbol{h}) \\
& \forall \boldsymbol{r} \text { on the orbit } r=r(\theta) \\
\leftrightarrow & r G M\left[1+e \cos \left(\theta-\theta_{p}\right)\right]=(\boldsymbol{r} \times \boldsymbol{v}) \cdot \boldsymbol{h}=h^{2} \\
& \text { on the points of the orbit } r=r(\theta) \\
\leftrightarrow & r(\theta) \equiv\left(P, e, \theta_{p}\right) .
\end{aligned}
$$

Infinite Set of LRL Vectors: Starting from $\boldsymbol{B}$ we may construct an infinite set of LRL vectors each of which plays the same role as $\boldsymbol{B}$. If $O$ is an arbitrary orthogonal matrix $(3 \times 3)$, then each vector $\boldsymbol{B}^{\prime}=O \boldsymbol{B}=$ $h O \boldsymbol{v}-G M O \boldsymbol{J}=e G M O \boldsymbol{J}_{p}$ is a constant of motion $\left(\mathrm{d} \boldsymbol{B}^{\prime} / \mathrm{d} t=O(\mathrm{~d} \boldsymbol{B} / \mathrm{d} t)=0\right)$. The equivalence

$$
\boldsymbol{B}=\boldsymbol{B}_{p} \leftrightarrow \boldsymbol{B}^{\prime}=O\left(e M G \boldsymbol{J}_{p}\right)
$$

shows that the same information concerning the orientation of an orbit is contained in an LRL vector as much in its transforms; the vector $O^{-1} \boldsymbol{B}^{\prime}$ is perpendicular to the symmetry axis of the orbit. By the properties of an orthogonal matrix we have $|O \boldsymbol{B}|=B=e G M$. To obtain the Equation of the orbit from $\boldsymbol{B}^{\prime}=O \boldsymbol{B}_{\boldsymbol{p}}$ we take the inner product of both sides by the vector $O \boldsymbol{J}$ :

$$
O \boldsymbol{J} \cdot O \boldsymbol{B}=e M G\left(O \boldsymbol{J} \cdot O \boldsymbol{J}_{p}\right) .
$$

Since the inner product is invariant under orthogonal transformations, we have

$$
\begin{aligned}
& \boldsymbol{J} \cdot \boldsymbol{B}=e M G\left(\boldsymbol{J} \cdot \boldsymbol{J}_{p}\right) \\
& \text { i.e. } \quad h r \dot{\theta}-G M=e M G \cos \left(\theta-\theta_{p}\right), \\
& \text { or } \quad h^{2} / r-G M=e M G \cos \left(\theta-\theta_{p}\right),
\end{aligned}
$$

which yields the orbit:

$$
r^{-1}=\left(G M / h^{2}\right)\left(1+e \cos \left(\theta-\theta_{p}\right)\right)
$$

Relative Velocity Hodographs: From (3.2) we have

$$
\boldsymbol{v}=(G M / h)\left(\boldsymbol{J}+e \boldsymbol{J}_{p}\right)
$$

which asserts that the velocity is $G M / h$ times the sum of a fixed vector $e \boldsymbol{J}_{p}$ of length $e$ and a vector $\boldsymbol{J}$ of fixed length 1 and variable direction. Thus the velocity vector depicts in the $v$-space a full circle for $e<1$, a circle less a point for $e=1$, and a proper circular arc for $e>1$ (Figure 2). The latter statements utilize the fact that the velocity's direction takes all possible values for elliptic orbits, constrained in the range $(0, \pi)$ for parabolic orbits, and in the range $\left(-\cos ^{-1}(-1 / e)+\pi, \cos ^{-1}(-1 / e)\right)$ for hyperbolic orbits.

For elliptic orbits the vector $v$ rotates on the circle with a period equal to the period of motion. For unbound motion the vector $v$ takes, starting from a point, an infinite time to reach an end of the circular arc. In hyperbolic motion, the circular arc tends to a circle less a point when $e$ tends to 1 , gets smaller with increasing values of $e$, and tends to a point when $e$ tends to infinity. The above simple method in obtaining the velocity hodographs refines Butikov's results [3] which were obtained in a lengthy manner for motion of a body in a central field.

\section{The Angular Momentum and Energy of an Orbit}

Inserting $\dot{r}_{p}=0$ and $h=r_{p} v_{p}$ in (1.2i) we get

$$
2 \varepsilon=v_{p}^{2}-\frac{2 G M}{r_{p}}=\frac{G M}{r_{p}}(e-1),
$$



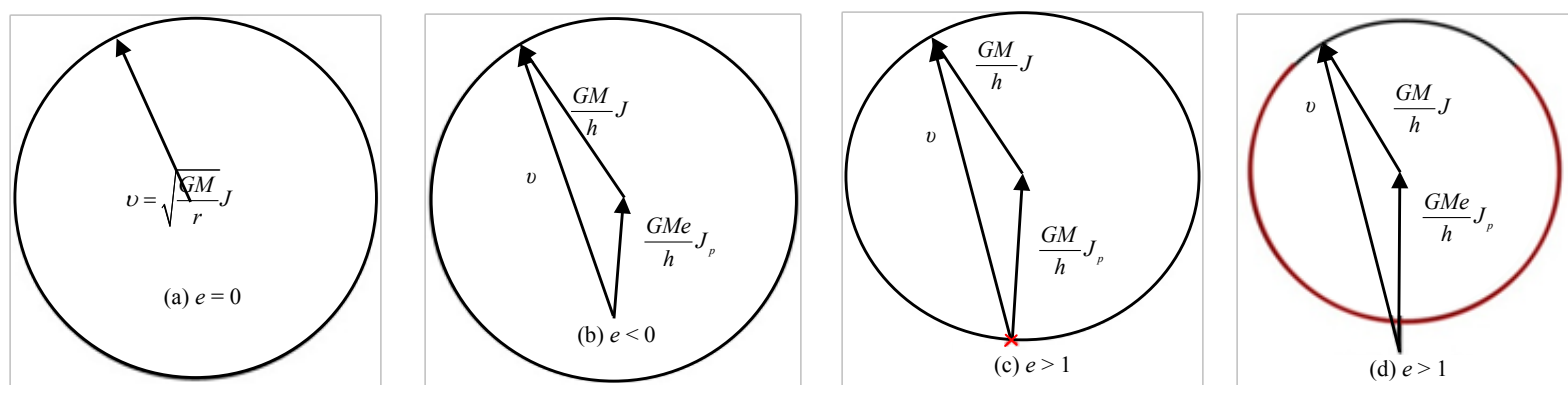

Figure 2. Hodographs of a gravitational 2-body system; they are (a) circles with radii $\sqrt{G M / r}$ for circular motion, (b) a full circle for elliptic motion, (c) a circle less a point for parabolic motion, and (d) a proper circular arc for hyperbolic motion. The radii of the hodographs in non-circular motions are $G M / h$.

where we have used (2.1ii) to write the last equality. Substituting $r_{p}=P /(1+e)$ in the latter relation yields

$$
\varepsilon=G M \frac{e^{2}-1}{2 P}= \pm \frac{G M}{2 a},(e \neq 1),
$$

where $a$ is the semi-major axis of the trajectory, and the $+(-)$ sign corresponds to hyperbolic (elliptic) orbits. We already know that $\varepsilon=0$ for parabolic orbit.

With $M$ is fixed, equivalent orbits are characterized by the same $(h, \varepsilon)$. Although a given relative orbit has specific values of angular momentum and energy reduced densities, there correspond to the same relative orbit different angular momenta $L=\mu h$ and energies $E=\mu \varepsilon$ depending on the distribution of the total mass $M$ between the two particles. By (4.2) the energies of the system when in an elliptic or hyperbolic orbit $\{P, e\}$ are

$$
E= \pm G m_{1} m_{2} / 2 a, \quad(e \neq 1)
$$

When the total mass is fixed, the reduced mass

$$
\mu=m_{2}\left(1-m_{2} / M\right)
$$

is an increasing function in $m_{2}$, with a minimum value $\mu=m_{2}$ for $m_{2} \ll M$, and a maximum value $\mu=M / 4=m / 2$ for $m_{2}=m_{1}=m$.

Thus the angular momentum and energy associated with the same relative orbit (and fixed $M$ ) may vary in absolute value from infinitesimal values corresponding to an infinitesimal reduced mass $\mu$ (i.e. infinitesimal $m_{2}$ ) to the maximum values:

$$
\begin{aligned}
& L_{\max }=M h / 4=M r_{p} v_{p} / 4 \\
& |E|_{\max }=M|\varepsilon| / 4=G M^{2} / 8 a
\end{aligned}
$$

corresponding to $m_{1}=m_{2}$. The latter Equation applies to elliptic and hyperbolic orbits; whereas the energy vanishes in case of a parabolic orbit.

\section{Laws of Equivalent Relative Orbits}

When we let, the perihelion unit vector $\boldsymbol{I}_{p}$, the rotation angle $\varphi$ about $\boldsymbol{I}_{p}$, and the center of mass (its coordinates in an inertial frame $S$ ) vary and take all their possible values while $r_{p}$ and $v_{p}$ are held constant, we obtain a 6-parameter family $\Gamma\left(\varphi, \boldsymbol{I}_{p}, \boldsymbol{C} \boldsymbol{C}^{\prime} \mid r_{p}, v_{p}\right)$ of equivalent orbits which result from one orbit $\left\{r_{p}, v_{p}\right\}$ through the action of orthogonal transformations [11]

$$
\boldsymbol{R}=\mathrm{O}+\boldsymbol{b},
$$

where $O$ is an arbitrary orthogonal $(3 \times 3)$ matrix and $\boldsymbol{b}$ is an arbitrary $(3 \times 1)$ vector signifying an arbitrary displacement $\boldsymbol{C} \boldsymbol{C}^{\prime}$ of the center of mass. Indeed, and because the space is homogeneous with respect to a closed two-body system, the latter remains equivalent to itself after a rotation, translation or inversion applied to it as a whole. 
Employing the 1 - 1 correspondences (2.4) we obtain the mass-independent LRL vector

$$
\boldsymbol{B}^{\prime}=\frac{\boldsymbol{B}}{M}=\frac{h}{M} \boldsymbol{v}-G \boldsymbol{J}=\bar{h} \boldsymbol{\vartheta}-G \boldsymbol{J}
$$

which is constant on an orbit with perihelion vector $r_{p} \boldsymbol{I}_{p}$ at the value $\boldsymbol{B}_{p}^{\prime}=e G \boldsymbol{J}_{p}$ whatever was the total mass.

The following laws [1] govern equivalent orbits with different total masses:

L1. Given $m_{1}$ and $r_{p}$, the same relative orbit occurs whether the mass of the second body is $m_{2}^{\prime}$ or $m_{2}$ provided the respective relative velocities are such that

$$
\left(m_{1}+m_{2}\right)^{-1 / 2} v_{p}=\left(m_{1}+m_{2}^{\prime}\right)^{-1 / 2} v_{p}^{\prime}
$$

L2. No matter how a fixed total mass $M$ is distributed between the two particles, the same relative trajectory occurs for the same initial conditions $\left(r_{p}, v_{p}\right)$.

For equivalent orbits with different total masses $M$ and $M^{\prime}$ we have $\bar{h}=\bar{h}^{\prime}, \bar{\varepsilon}=\bar{\varepsilon}^{\prime}$, and hence

\section{L3. The Law of Areal Velocity:}

$$
h / \sqrt{M}=h^{\prime} / \sqrt{M^{\prime}}=\sqrt{G P} .
$$

\section{L4. The Law of Orbit's Energy}

$$
\frac{\varepsilon}{M}=\frac{\varepsilon^{\prime}}{M^{\prime}}= \pm \frac{G}{2 a}(e \neq 1) .
$$

But since $E=\mu \varepsilon, E^{\prime}=\mu^{\prime} \varepsilon^{\prime}$ we have

$$
E / m_{1} m_{2}=E^{\prime} / m_{1}^{\prime} m_{2}^{\prime} .
$$

From the last equation and from Newton's law in gravitation we have

$$
F / F^{\prime}=E / E^{\prime}=m_{1} m_{2} / m_{1}^{\prime} m_{2}^{\prime},(e \neq 1),
$$

where $F>0$ is the length of the force vector at $(r(\theta), \theta)$. The relation (5.6) shows that the energy of a system with a total mass $M^{\prime}=m_{1}^{\prime}+m_{2}^{\prime}<M=m_{1}+m_{2}$ can exceed the energy of a system of total mass $M$, occupying an equivalent orbit, if $m_{2}$ was sufficiently small while both $m_{1}^{\prime}$ and $m_{2}^{\prime}$ are finite, say $m_{1}^{\prime} \approx m_{2}^{\prime}$. By (5.7), the same statement applies to the corresponding attractive forces.

Inserting the expression of $F$ at a distance $r$ in the relation $F / E=F^{\prime} / E^{\prime}$ we obtain

$$
E / F=E^{\prime} / F^{\prime}= \pm r^{2} / 2 a(e \neq 1)
$$

From the latter relation we obtain the obvious relation

$$
F=2 a|E| / r^{2}(e \neq 1)
$$

which could also have been obtained through expressing $G m_{1} m_{2}$ in Newton gravitational law in terms of the orbit's energy (4.3).

L5. Law of Periods: Because two equivalent bound orbits have the same area $A$, which equals to the product of the period by the areal velocity, we have

$$
h \tau=h^{\prime} \tau^{\prime}=2 A .
$$

Dividing the latter equations side to side by (5.4) we obtain

$$
\tau \sqrt{M}=\tau^{\prime} \sqrt{M^{\prime}}=2 \pi a^{3 / 2} / \sqrt{G},
$$

which states: the periods of motions in equivalent orbits are inversely proportional to the square root of the corresponding total masses. If the mass of Jupiter, which is about 1000 times of the Earth's mass, was just equal to the Earth's mass then its period, which is about 12 years, would increase by about 50 hours.

Two systems of total masses $M$ and $M^{\prime}$ can have equivalent trajectories if $\left(r_{p}, \vartheta_{p}\right)=\left(r_{p}^{\prime}, \vartheta_{p}^{\prime}\right)$. It follows that, for equivalent orbits 


$$
v_{p} / \sqrt{M}=v_{p}^{\prime} / \sqrt{M^{\prime}} .
$$

Denote the points of two equivalent orbits $\left\{r_{p}, \vartheta_{p}\right\}$ corresponding to total masses $M$ and $M^{\prime}$ by $(r, \theta)$ and $\left(r^{\prime}, \theta^{\prime}\right)$ respectively, and take $\theta_{p}=\theta_{p^{\prime}}^{\prime}$.

L6. The Law of Angular Velocity: For $\theta=\theta^{\prime}$, we have $r=r^{\prime}$, and by (5.4),

$$
\dot{\theta} / \sqrt{M}=\dot{\theta}^{\prime} / \sqrt{M^{\prime}}\left(\text { for } \theta=\theta^{\prime}\right)
$$

L7. The Law of Velocities: The relative velocities associated with the two equivalent orbits are

$$
\boldsymbol{v}=\left(\frac{\mathrm{d} r}{\mathrm{~d} \theta} \boldsymbol{I}+r \boldsymbol{J}\right) \dot{\theta}, \boldsymbol{v}^{\prime}=\left(\frac{\mathrm{d} r^{\prime}}{\mathrm{d} \theta^{\prime}} \boldsymbol{I}^{\prime}+r^{\prime} \boldsymbol{J}^{\prime}\right) \dot{\theta}^{\prime}
$$

The parenthesized quantities in the latter two equations are equal in magnitude for $\theta=\theta^{\prime}$, because $r=r^{\prime}$ and $\mathrm{d} r / \mathrm{d} \theta=\mathrm{d} r^{\prime} / \mathrm{d} \theta^{\prime}$. It follows that

$$
v / \dot{\theta}=v^{\prime} / \dot{\theta}^{\prime} \quad\left(\text { for } \theta=\theta^{\prime}\right)
$$

Multiplying the latter equation side to side by equation (5.13) yields

$$
v / \sqrt{M}=v^{\prime} / \sqrt{M^{\prime}}\left(\text { for } \theta=\theta^{\prime}\right)
$$

It states: the relative velocity of the system in a given orbit is proportional to the square root of its mass.

L8. The Law of Radial Velocity: Squaring both side of (5.15) yields for $\theta=\theta^{\prime}$

$$
M^{-1}\left(\dot{r}^{2}+r^{2} \dot{\theta}^{2}\right)=M^{\prime-1}\left(\dot{r}^{\prime 2}+r^{\prime 2} \dot{\theta}^{\prime 2}\right)
$$

By (5.13) and since $r=r^{\prime}$ for $\theta=\theta^{\prime}$ we have

$$
\dot{r} / \sqrt{M}=\dot{r}^{\prime} / \sqrt{M^{\prime}} \text { for } \theta=\theta^{\prime} .
$$

L9. Law of Relative Acceleration: From (1.1), we have

$$
\boldsymbol{a} / M=\boldsymbol{a}^{\prime} / M^{\prime}=-G \boldsymbol{I} / r^{2} \text { for } \theta=\theta^{\prime} .
$$

L10. Relative Areal Velocity: The relation

$$
h=r^{2} \dot{\theta}=\left(r_{1}+r_{2}\right)^{2} \dot{\theta}=\left(\sqrt{h_{1}}+\sqrt{h_{2}}\right)^{2},
$$

determines the areal velocity in terms of the areal velocities of the two particles in $S_{c}$. For $m_{1}=m_{2}, h_{1}=h_{2}$ and $h=4 h_{2}$. When $m_{1}$ is dominant, $h \approx h_{2}$.

L11. Angular Momenta of the System and its Parts: The angular momentum of the system is the sum of the angular momenta of the constituent particles: $\boldsymbol{L}=\boldsymbol{l}_{1}+\boldsymbol{l}_{2}$. It follows that the magnitudes of these angular momenta satisfy the relation

$$
L=l_{1}+l_{2}=m_{1} h_{1}+m_{2} h_{2}
$$

in which $h_{i}=r_{i}^{2} \dot{\theta},(i=1,2)$. An expression equivalent to (5.19) can be obtained from (5.18) and from the facts, $L=\mu h, l_{i}=m_{i} h_{i}(i=1,2)$ :

$$
\sqrt{L / \mu}=\sqrt{l_{1} / m_{1}}+\sqrt{l_{2} / m_{2}} \text {. }
$$

When $m_{1}$ is dominant we have $L \approx l_{2}$, and when $m_{1}=m_{2}$, we have $l_{1}=l_{2}$ and $L=2 l_{2}$.

The laws of equivalent orbits can be deduced from (1.4) which shows that two orbits with different total masses are equivalent provided that $h^{2} / M$ and $\varepsilon h^{2} / M^{2}$ are the same for both orbits. The latter conditions are equivalent to

$$
h / \sqrt{M}=h^{\prime} / \sqrt{M^{\prime}} \text { and } \varepsilon / M=\varepsilon^{\prime} / M^{\prime} .
$$

Since $r(\theta)$ is the same for equivalent orbits we deduce from the relations (5.21) and (1.2) written in the form 


$$
\frac{2 \varepsilon}{M}=\frac{\dot{r}^{2}}{M}+\frac{h^{2}}{M r^{2}}-\frac{2 G}{r}, \frac{h}{\sqrt{M}}=r^{2} \frac{\dot{\theta}}{\sqrt{M}}
$$

that

$$
\dot{r} / \sqrt{M}=\dot{r}^{\prime} / \sqrt{M^{\prime}} \text { and } \dot{\theta} / \sqrt{M}=\dot{\theta}^{\prime} / \sqrt{M^{\prime}} .
$$

Hence $v / \sqrt{M}=v^{\prime} / \sqrt{M^{\prime}}$, and consequently $\tau \sqrt{M}=\tau^{\prime} \sqrt{M^{\prime}}$.

\section{Revision of Galileo's Law of Free Fall}

We discuss here the fall of two bodies towards each other in a straight line. Straight motion is realized if and only if $\dot{\theta}=0$. In this case $h=0$, and by (1.2)

$$
\varepsilon=2 E / \mu=\dot{r}^{2}-2 G M / r .
$$

The latter relation shows that the two bodies recede from each other from a point $r$ to infinity if their relative velocity $\dot{r}$ is such that $\dot{r} \geq \sqrt{2 G M / r}$; they reach infinity with zero velocity when the equality sign holds.

For the initial conditions (at $\left.t=0, r=r_{0} \& \dot{r}=0\right), \varepsilon=-2 G M / r_{0}$ and by (6.1),

$$
\dot{r}^{2}(t)=2 G M\left(\frac{1}{r}-\frac{1}{r_{0}}\right),
$$

or

$$
\frac{\mathrm{d} r}{\mathrm{~d} t}=-\sqrt{\frac{2 G M}{r_{0}}} \sqrt{\frac{r_{0}-r}{r}} .
$$

Separating the variables and integrating we obtain

$$
t=\sqrt{\frac{r_{0}}{2 G M}}\left\{\sqrt{r\left(r_{0}-r\right)}+r_{0} \sin ^{-1} \sqrt{\frac{r_{0}-r}{r_{0}}}\right\},
$$

which shows that the duration of falling from an initial altitude $r_{0}$ to a final altitude $r$ is inversely proportional to the square root of the total mass $M$.

The duration of the complete fall of two particles towards each other starting from an initial separation $r_{0}$ is

$$
t(r \rightarrow 0)=\pi r_{0}^{3 / 2} / 2 \sqrt{2 G M} .
$$

The invalidity of GLFF in general is apparent from (1.1) which shows that the acceleration acquired by a mass $m_{2}^{\prime}$ falling freely from rest due the attraction of $m_{1}$ is $a^{\prime}=-G M^{\prime} / r^{\prime 2}$, where $r^{\prime}$ is its distance from $m_{1}$, and $M^{\prime}=m_{1}+m_{2}^{\prime}$. It follows that at the same distance $r=r^{\prime}$ from $m_{1}$ the relative acceleration is proportional to the total mass:

$$
a / M=a^{\prime} / M^{\prime} .
$$

By (6.3) the velocity of a body falling freely from the altitude $r_{0}$ is proportional to the square root of the total mass

$$
\frac{v}{\sqrt{M}}=\frac{v^{\prime}}{\sqrt{M^{\prime}}} \text { for } r=r^{\prime} .
$$

From (6.4) we obtain the revised GLFF; it states that the duration of a free fall from $r_{0}$ by a distance $h=r_{0}-r$ is inversely proportional to the square root of the total mass:

$$
t \sqrt{M}=t^{\prime} \sqrt{M^{\prime}} .
$$

When the free fall covers only a small distance $h=r_{0}-r \ll r$, the following approximations hold

$$
r_{0} \approx r, \quad \sqrt{r_{0}} \sqrt{r\left(r_{0}-r\right)} \approx r \sqrt{h}
$$




$$
\sqrt{r_{0}} r_{0} \sin ^{-1} \sqrt{\frac{r_{0}-r}{r_{0}}} \approx \sqrt{r_{0}} r_{0} \cdot \sqrt{\frac{r_{0}-r}{r_{0}}}=r \sqrt{h} .
$$

Substituting in (6.4) we obtain

$$
t \approx 2 r \sqrt{h} / \sqrt{2 G M} .
$$

Solving for $h$ we find the familiar expression,

$$
h=\frac{1}{2} \frac{G M}{r^{2}} t^{2}=\frac{1}{2} a t^{2},
$$

where we used (1.1) to set $G M / r^{2}=a$, which is approximately constant over a small change $h$ in $r$.

\section{The Approximation of a Central Gravitational Field}

We discuss here the effect of the smaller mass' value $m_{2}$ on the duration of a fall towards a given (constant) mass $m_{1}$ in case of a straight trajectory, or on its period of rotation about $m_{1}$ in a bound orbit. According to the value of $m_{2}$ three cases are distinguished:

(i) The mass $m_{2}$ is infinitesimally small.

(ii) The mass $m_{1}$ is dominant $\left(m_{1} \gg m_{2}\right)$, in which case

$$
\begin{aligned}
& m_{1} \approx M, \boldsymbol{r}_{1}=-\frac{m_{2}}{M} \boldsymbol{r} \approx 0, \boldsymbol{r}_{2}=\frac{m_{1}}{M} \boldsymbol{r} \approx \boldsymbol{r} \\
& \boldsymbol{v}_{1} \approx 0, \boldsymbol{v}_{2} \approx \boldsymbol{v}, \boldsymbol{a}_{1} \approx 0, \boldsymbol{a}_{2} \approx \boldsymbol{a} .
\end{aligned}
$$

where as the ratio $m_{2} / m_{1}$ is small but finite in case (ii), it is infinitesimally small in case (i), and hence the approximations (7.1) hold effectively as equalities in case (i).

(iii) The masses of the particles are equal $\left(m_{1}=m_{2}=m\right)$. Here we have

$$
\begin{aligned}
& \mu=\frac{1}{2} m, \quad r_{1}=r_{2}=\frac{1}{2} r, \\
& -\boldsymbol{v}_{1}=\boldsymbol{v}_{2}=\frac{1}{2} \boldsymbol{v}, \quad-\boldsymbol{a}_{1}=\boldsymbol{a}_{2}=\frac{1}{2} \boldsymbol{a} .
\end{aligned}
$$

(A) Straight Trajectory: The free fall's duration given by (6.3) can be written as

$$
t=T_{n} / \sqrt{1+m_{2} / m_{1}},
$$

where

$$
T_{n}=\sqrt{\frac{r_{0}}{2 G m_{1}}}\left\{\sqrt{r\left(r_{0}-r\right)}+r_{0} \sin ^{-1} \sqrt{\frac{r_{0}-r}{r_{0}}}\right\},
$$

is the free fall duration of a minute object with a negligible mass, $n$, in comparison with $m_{1}$, call it "the nothing". Thus the nothing in this context is an infinitesimal mass $n$ with the property, $T_{n}\left(n / m_{1}\right)$ is negligible. In fact, it is the fall's time $T_{n}$ of the nothing, what corresponds to GLFF; it results from $t$ in (7.3) on letting $m_{2} \rightarrow 0$.

Equation (7.3) reduces to

and to

$$
t=T_{n} / \sqrt{2} \text { for } m_{1}=m_{2}
$$

$$
t \approx\left(1-\frac{m_{2}}{2 m_{1}}\right) T_{n} \text { for } m_{1} \gg m_{2} .
$$

Equation (7.6) shows that the free fall's time $t$ of a mass $m_{2}$, with $m_{2} / m_{1} \ll 1$, is less than the $n$ fall's time by $\left(m_{2} / 2 m_{1}\right) T_{n}$, which is directly proportional to its mass with a proportionality constant $1 / 2 m_{1}$. 


\section{Example: Free Fall towards the Earth.}

We consider here the free fall corresponding to the gravitational force between the Earth and objects that have much smaller masses. We neglect of course the effect of air resistance. With the earth's mass: $m_{1} \approx 6 \times 10^{24} \mathrm{~kg}$, the masses of a hammer or a feather can be counted as the nothing. Corresponding to the fall duration $T_{n}=100$ seconds of the nothing, the fall time of a mass $m_{2}$ falling freely to earth from the same point at which the nothing starts will be

$$
t=\left(1-\frac{m_{2}}{2 m_{1}}\right) T_{n}=\left(1-\frac{m_{2} \mathrm{~kg}}{12 \times 10^{24} \mathrm{~kg}}\right) \times 100 \mathrm{sec} .
$$

For a difference $\left(m_{2} / 2 m_{1}\right) T_{n}$ not exceeding $10^{-19}$ seconds we have to choose the falling mass such that

$$
\frac{m_{2}}{2 m_{1}} T_{n}=\frac{m_{2}}{12 \times 10^{24}} \times 100 \mathrm{sec} \leq 10^{-19} \text { seconds, }
$$

which yields: $m_{2} \leq 12 \times 10^{3} \mathrm{~kg}=12$ Ton. That is, the falling body with mass not exceeding 12 Ton will advance $n$ in a fall trip of duration 100 seconds by less than $10^{-10} \mathrm{~ns}$. The advance period for a body of mass $120 \mathrm{~kg}$ will be $10^{-12} \mathrm{~ns}$, and if the fall time is only 5 seconds $(\approx 122.5$ meters height) then the advance period will be about $5 \times 10^{-14} \mathrm{~ns}$, during which light travels only $15 \times 10^{-5}$ Angestrom.

On the other hand, and if Earth and Venus, that have roughly equal masses, fall freely towards each other (in the absence of the sun and other planets) then there corresponds to $T_{n}=100$ seconds the fall's period $t=T_{n} / \sqrt{2} \approx 70$ seconds.

(B) Bound Motion: Setting $M^{\prime}=m_{1}$ in (5.11) gives the nothing's orbit's period $\tau_{n}=\tau^{\prime}$ in the field of the body $m_{1}$,

$$
\tau_{n}=2 \pi a^{3 / 2} / \sqrt{G m_{1}} .
$$

The same Equation (5.11) yields the orbital period $\tau$ of a mass $M=m_{1}+m_{2}$ tracing the same nothing's orbit by:

$$
\tau=\tau_{n} / \sqrt{1+m_{2} / m_{1}}
$$

The period (7.8) reduces to

$$
\begin{aligned}
& \tau=\tau_{n} \text { for the } n, \\
& \tau \approx\left(1-m_{2} / 2 m_{1}\right) \tau_{n} \text { for } m_{2} \ll m_{1}, \\
& \tau=\tau_{n} / \sqrt{2} \text { for } m_{2}=m_{1} .
\end{aligned}
$$

It is noted that the relations (7.8-7.11) are valid only for equivalent orbits, and consequently the corresponding relative velocity depends, by (5.15), on the mass $m_{2}$ through the relation

$$
v=\sqrt{1+m_{2} / m_{1}} v_{n},
$$

with the nothing velocity at the perihelion is constrained by

$$
\sqrt{G m_{1} / r_{p}} \leq v_{n p}<\sqrt{2 G m_{1} / r_{p}} .
$$

In particular $v=\sqrt{2} v_{n}$ for $m_{2}=m_{1}$.

The relation (7.8) shows that the period $\tau$ of a bound motion of a mass $m_{2}$ is less than the corresponding period $\tau_{n}$ of the nothing by the factor appearing on its right hand-side. Because this factor decreases when $m_{2}$ increases, the heavier the body the shorter period it has.

We finally mention that the mass $n$, which is not zero, has no intrinsic value; its values belong to a range that depends on the dominant mass and the degree of accuracy sought, or within which is the resolution power of our equipments. 


\section{Perturbation of the Nothing's Orbits}

It is clear that a finite mass $m_{2}$ perturbs the gravitational field generated by the massive body $m_{1}$. To quantify the order of perturbation, we revert to equation (2.1) and specify the relative orbit by the ordered pair:

$$
(P, e+1)=\frac{1}{1+m_{2} / m_{1}} \frac{v_{p}^{2}}{G m_{1}}\left(r_{p}^{2}, r_{p}\right) .
$$

Letting $m_{2} \rightarrow 0$ we obtain the $n$ orbit, $\left(P_{n}, e_{n}\right)$, in the gravitational field of $m_{1}$ :

$$
\left(P_{n}, e_{n}+1\right)=\frac{v_{n p}^{2}}{G m_{1}}\left(r_{p}^{2}, r_{p}\right) \text {. }
$$

It follows from (8.2) that, given a mass $m_{1}$, then for each fixed value of $r_{p}$ there exists an one-to-one correspondence between each value of $v_{n p}$ in the range $v_{n p}>\sqrt{G m_{1} / r_{p}}$ and an orbit $\left(P_{n}, e_{n}\right)$ of the nothing. Thus when $r_{p}$ is given, a nothing's orbit is determined solely by $v_{n p}$. Equivalently, the orbits of the nothing are uniquely determined by its initial conditions.

We discuss here the perturbation in the $n$ orbit in the gravitational field of a mass $m_{1}$ when $n$ is replaced by a finite mass $m_{2}$. On fixing $r_{p}$ the orbit of the system is determined by $v_{p}$ and $m_{2}$. We already know that the same nothing's orbit $\left(P_{n}, e_{n}\right)$ is maintained for $m_{2}$ if its velocity $v_{p}$ is such that

$$
v_{p}=v_{n p} \sqrt{1+m_{2} / m_{1}}
$$

If we replace $n$ by $m_{2}$ and keep $\left(r_{p}, v_{p}\right)=\left(r_{n p}, v_{n p}\right)$, the orbit $\left(P_{n}, e_{n}\right)$ of $n$ will change to the orbit $(P, e)$ given by (8.1). We determine here the element of the new orbit in terms of the $n$ orbit and $m_{2}$. For brevity we set $\beta=m_{2} / m_{1}$. From (8.1),

$$
P=\frac{P_{n}}{1+\beta}, e=\frac{e_{n}-\beta}{1+\beta}
$$

The latter equations show that the eccentricity and the semi-latus rectum of the new orbit decreases with an increasing mass $m_{2}$. Direct consequences of the last statement are the following:

C1. Since $P<P_{n}$ and $e<e_{n}$, the new orbit can be qualitatively different, in the sense that an unbound motion may be converted to a bound one. Indeed, by (8.4),

$$
e<1 \leftrightarrow e_{n}<1+2 \beta
$$

If in particular the n's orbit is unbound with $1 \leq e_{n}<1+2 \beta$, then the mass $m_{2}$ with the initial conditions $\left(r_{p}, v_{p}\right)=\left(r_{n p}, v_{n p}\right)$ will trace a bound orbit.

C2. An increase in mass leaves bound orbits bound, provided the lower constraint (2.2ii) on velocity remains valid. In particular the new orbit is circular if $e_{n}=\beta$.

C3. A curious case occurs for the nothing's circular orbits. As implied by (8.4), the replacement of the nothing when in a circular orbit $\left(\right.$ i.e. $\left.e_{n}=0\right)$ with minute mass results in the absurd, a negative eccentricity. This implies that no material body can be in any of the nothing's circular orbits with the same initial conditions. Or as to say, the $n$ circular orbits do not exist physically. The latter results is well justified on recalling that the nothing is merely a mathematical abstraction of a small mass. It is clear of course that the $n$ 's circular orbits can be occupied by material bodies but with velocities determined by (8.3).

The semi-major axis and the period of the new orbit are easily found to be

$$
\begin{aligned}
& a=a_{n}(1+\beta) /\left[1+2 \beta /\left(1-e_{n}\right)\right], \\
& \tau=\tau_{n}(1+\beta)\left[1+2 \beta /\left(1-e_{n}\right)\right]^{-3 / 2} .
\end{aligned}
$$

To the first order in $\beta$ we have

$$
a=a_{n}\left[1-\beta\left(e_{n}+1\right) /\left(1-e_{n}\right)\right]
$$




$$
\tau=\tau_{n}\left[1-\beta\left(2+e_{n}\right) /\left(1-e_{n}\right)\right]
$$

From (8.1) and (8.4), we deduce the following relation

$$
\boldsymbol{r}_{n}=\boldsymbol{r}(1+\beta)\left[1+\beta \frac{(e+1) \cos \theta}{1+e \cos \theta}\right]^{-1}
$$

which shows that the $n$ orbits are at larger distances from that at which $m_{2}$ orbits $m_{1}$. Equality holds for $\theta=0$ or $\theta=\pi$.

\section{Discrepancies between the Equivalence Principle and Newton's Theory}

Newton's laws of motion, which hold in inertial frames, form one whole entity by which the motion of a system can be specified. The newly introduced physical entity by Newton, "the force", was not specified by his second law $\boldsymbol{f}=m_{2} \boldsymbol{a}_{2}$ alone, but also by the supplementary law of action and reaction, $\boldsymbol{f}_{12}+\boldsymbol{f}_{21}=0$. For a closed system of $N$ free particles, $\sum_{i=1}^{N} m_{i} \boldsymbol{a}_{i}=0$, and the force exerted on a particle $N$ is $\boldsymbol{f}_{N}=m_{N} \boldsymbol{a}_{N}=-\sum_{j=1}^{N-1} \boldsymbol{f}_{j}$, where $\boldsymbol{f}_{j}$ is the force exerted on $m_{j}$ by $m_{N}$. Although it was not stated explicitly, the concept, "force", was coined to refer to interactions between particles, or systems of such. Newton's second law specifies the acceleration of a body of mass $m_{N}$ in any inertial frame $S$, while the motions of the remaining bodies in the closed system are pragmatically overlooked.

For a closed system of $N$ particles that interact mutually trough gravitational forces, the acceleration $\boldsymbol{a}_{N}$ of the $N$-th particle,

$$
\boldsymbol{a}_{N}=\frac{\boldsymbol{f}}{m}=-G \sum_{j=1}^{N-1} m_{j}\left(\boldsymbol{r}-\boldsymbol{r}_{j}\right) /\left|\boldsymbol{r}-\boldsymbol{r}_{j}\right|^{3},
$$

where $m$ is its mass, $\boldsymbol{r}$ is its position vector, and $\boldsymbol{r}_{j}$ is the position vector of the $j$-th particle, is independent of its mass but depends on its position and the masses and positions of the remaining particles $\operatorname{Rem}=\left\{m_{j}, j=1, \cdots, N-1\right\}$. It is important to note that $\boldsymbol{a}_{N}$ is the absolute acceleration of $m$; it is its acceleration relative to the $C M$ frame (or to any other inertial frame). However, only when the mass $m$ is negligible in comparison with the total mass of Rem (i.e., $\left.m \ll \sum_{i=1}^{N-1} m_{i}\right)$, the center of mass $C M$ can be identified with the center of mass $C M^{\prime}$ of Rem, and the absolute acceleration $\boldsymbol{a}_{N}$ will be identical to the acceleration $\boldsymbol{a}$ relative to $C M^{\prime}$. In the latter case we can speak of a time-dependent acceleration field, or a gravitational field, $\boldsymbol{a}(\boldsymbol{r}, t)$, generated by $\operatorname{Rem}$ and defined at each point $\boldsymbol{r}$ by the right hand-side of (9.1); it changes with time because the elements of Rem are moving.

For a 2-body system $(N=2)$, the concept of a gravitational field can be substantiated only when one mass, say $m_{1}$, is dominant, in which case the system's center of mass can be identified with the center of $m_{1}$ and considered stationary in $S_{c}$ and thus the system's total mass can be approximated by $m_{1}$. In this case a stationary gravita- tional field $\boldsymbol{a}$ set up by the dominant mass $m_{1}$ can be defined throughout the space by

$$
\boldsymbol{a}=-G m_{1} \boldsymbol{I} / r^{2}
$$

where $\boldsymbol{r}=r \boldsymbol{I}$ is the position vector of a point relative to the center of mass $m_{1}$. A body of a negligible mass placed at a position $\boldsymbol{r}$ acquires an absolute acceleration $\boldsymbol{a}$, and its motion is determined indeed by its initial conditions and does not depend on its mass.

The equivalence principle (EP), on which the general theory of relativity (GR) hinges, is based on the fact that the acceleration, inherent to inertial forces (centrifugal, Coriolis, $\cdots$ ) and to locally uniform gravitational forces, is independent of the mass of the body acted on by these forces. Therefore, the properties of motion in a uniformly accelerated frame of reference and in a uniform gravitational field are the same. To express it in a different way, no mechanical experiment carried out inside a laboratory can tell whether the laboratory is at rest on the earth's surface or moving far away from matter at a constant acceleration $\boldsymbol{g}$, where $\boldsymbol{g}$ is the acceleration due to earth's gravity at its surface. The latter fact was elevated by Einstein to a level of a principle [10-14], which states that: all laws of physics are the same in a uniform gravitational field and in a uniformly accelerating frame of reference. More general: gravitational fields and inertial fields are equivalent with respect to physics laws. During a short period of time the gravitational field subtended in a freely falling chamber can be considered uniform, and cancels 
out with the fictitious acceleration field in the falling chamber, and thus the motion inside the chamber is identical to it in an inertial frame [10-14]. Indeed, particles that are not subjected to forces remain at rest or move at constant velocities relative to the chamber. Since our article is concerned with motion under gravity we shall restrict the EP to its weak from, the mechanical meaning.

We explore here the domain of applicability of the EP and highlight the deviations of Newtonian predictions from that of the EP and GR in general. We shall also show that Newton's law of gravitation, which imposes no restriction on the interacting masses, is not truly a limiting case of Einstein field Equations, as stated in most textbooks [10-13], unless the approximation of central gravitational field holds. Indeed,

- The central gravitational field contained in a falling chamber deviates slightly from uniformity, which results in tidal forces $[10,12]$ by which particles on the same horizontal level approach each other and particles on the same vertical line recede from each other.

- Moreover, and as it was explained in earlier sections, heavier particles acquire greater acceleration. Treating the chamber containing freely falling particles as one rigid body of a mass much greater than its contents, results in the chamber falling faster than the particles inside. Thus the particles leave gradually the chamber's ceiling in the course of time, with lighter particles leaving faster than heavier ones. The latter effect can be attributed, in the chamber frame, to a weak force that decreases when the mass of the falling particle increases. It may worth to investigate if this force has any effect on comets tails, pointing away from the Sun, and which is mainly due to solar wind. The same weak force may have an effect on the relative motion of the system, Earth-Moon, considered as a chamber falling in an elliptic orbit round the Sun.

- We have seen that the relative acceleration between two bodies depend on the sum of their masses, with no distinction between an active and a passive mass (Equation (1.1)). The EP looks on the force exerted by a massive body $m_{1}$ on a test mass $m_{2}$ as being mediated by a gravitational field $\boldsymbol{g}(r)=-G m_{1} \boldsymbol{I} / r^{2}$ generated by $m_{1}$, with $\boldsymbol{f}=m_{2} \boldsymbol{g}(r)$. The interaction of two masses of the same order of magnitude is outside the do- main of the EP. In fact, there is no known exact solution for the two-body problem in GR [13].

In GR [10-15], the distribution of matter and energy in the space shapes the geometry of spacetime, which ceases to be a flat Minkowski space, but becomes instead a curved 4-dimensional pseudo Riemannian space [16], which is Minkowskian in the infinitesimal (i.e. in an infinitesimal open neighborhood of each point). More precisely, the metric tensor in the curved 4-space is linked to the energy-momentum tensor by Einstein's field Equations [10-15]. In this curved 4-space, test masses follow geodesics while light follows null geodesics. Each geodesic is determined by an initial position and instant of time together with an initial vector velocity. Each given set of initial conditions determines a unique geodesic, namely that which passes through the given 4-point in the direction specified by the particle initial velocity. In this formalism, the gravitational forces have been foregone with and absorbed in the metric. In fact, if the metric of the curved spacetime in the presence of a given mass-energy distribution is known then the geodesics of the curved 4-space can be determined [10-15], and it is an equivalent alternative to solving Einstein's field Equations.

We are now at a position to state what GR says about the results we have obtained using Newton's mechanics. We have seen that each initial condition $\left(r_{p}, v_{p}\right)$ determines an orbit $\left(p_{n}, e_{n}\right)$ of the nothing $n$. According to GR the same initial conditions yield the same orbit even if $n$ was replaced by a test mass $m_{2}$, with no clear cut between "small" and "large". In fact, the test mass can be as large as a planet of the solar system in the gravitational field of the Sun [10-15]. This however is not so in Newton's mechanics by which, upon fixing the initial conditions and increasing $n$ to $m_{2}$, the orbit becomes $(p, e)$ given by (8.1). As evidenced by (8.7), the perturbation involves also the motion's period when it is bound. The quantitative change in the period of Jupiter, had its mass been replaced by the Earth's mass, was already given as an example. We showed also in (8.C1) that for some specific range of initial conditions this perturbation in orbit can appear qualitatively where an unbound motion becomes bound. Conversely, the same orbit in GR cannot be obtained by two different sets of initial conditions. In Newton's mechanics, the same orbit is realized for the nothing and a particle of a finite mass $m_{2}$ provided the initial velocities for the same $r_{p}$ satisfy Equation (8.3).

For a weak static gravitational field generated by a body of mass $m_{1}$, the Einstein's field Equations yield in the non-relativistic limit the gravitational field $[11,12]$

$$
\boldsymbol{g}=-G m_{1} \boldsymbol{I} / r^{2}
$$

in which $\boldsymbol{g}$ is the acceleration acquired by a body when placed at a distance $r$ from the center of $m_{1}$ (and outside it) regardless of its mass $m_{2}$; it is the acceleration of $m_{2}$ relative to $m_{1}$, i.e. the acceleration in a frame $s_{1}$ co-moving with $m_{1}$. Confining discussion to 2-body problem, and recalling that only when $s_{1}$ is inertial, the 
force acting on a mass $m_{2}$ is its product by $\boldsymbol{g}$, we brief here the points of agreement and disagreements with Newtonian predictions:

1) Only when $\boldsymbol{g}$ can be identified with the absolute acceleration, which is realized for a negligible mass $m_{2}$ and to the extent that $m_{1}$ is effectively the center of mass, the force exerted on $m_{2}$ would be

$\boldsymbol{f}=m_{2} \boldsymbol{g}=-G m_{1} m_{2} \boldsymbol{I} / r^{2}$, which is Newton's law in gravitation. Thus: the latter agreement with Newtonian gravity is valid only when the conditions necessary for a meaningful definition of the gravitational field are fulfilled. In this case however, the field expression (9.3) is a limiting case of the Newtonian formula (1.1) corresponding to $M \approx m_{1}$.

2) If $m_{2}$ is not negligible then $\boldsymbol{g}$ is an erroneous expression for the relative acceleration $\boldsymbol{a}$ as given by (1.1). In fact we have seen [1] that the Newtonian gravitational force between the two bodies is $\boldsymbol{f}=\boldsymbol{\mu a}$. The coincidental agreement of $m_{2} \boldsymbol{g}$ with Newton's gravitational force is due to the fact that $\boldsymbol{g}$, which is the relative acceleration according to GR, coincides with the absolute acceleration of $m_{2}$, as is evidenced from Equation (9.1) for $N=2$.

Observation of binary stars should judge whether the relative acceleration is $\boldsymbol{g}$ or $\boldsymbol{a}$. In fact, measuring the system's period, semi-latus rectum (or eccentricity), and major axis determines the total mass, the orbit, and hence the acceleration.

\section{Conclusion}

An LRL vector which coincides with the vector velocity hodograph of a gravitational 2-body system was derived. It was shown that corresponding to elliptic, parabolic, or hyperbolic orbits the hodograph is a full circle, a circle less a point, a proper arc of a circle respectively. The conditions under which the concept of a gravitational field in Newtonian mechanics becomes meaningful were discussed, and the related mathematical concept of "the nothing" was introduced. The perturbations in the nothing's orbits due to a finite mass were determined, and it was shown that the nothing's circular orbits don't exist physically in the sense that they cannot be occupied by any mass with the same initial condition. The equivalence principle is in accord with Newtonian mechanics only for nothing-like masses, whereas basic contradictive predictions emerge for non-negligible test masses. It is also shown that Newton's law in gravitation, which applies to arbitrary masses, is not an approximating limit to Einstein's field Equation in case of static weak fields, but that both theories have the Newtonian gravitational field as a common approximating limit.

\section{REFERENCES}

[1] C. P. Viazminsky and P. Vizminiska, "On the Gravitational Two-Body System and an Infinite Set of Laplace-Runge-Lenz Vectors," Applied Mathematics, Vol. 4, No. 5, 2013, pp. 774-784.

[2] A. Alemi, "Laplace-Runge-Lenz Vector," 2009. www.cds.Caltech.edu/Wiki/Alemicds205final.pdf

[3] E. L. Butikov, "The Velocity Hodograph for Arbitrary Keplerian Motion,” European Journal of Physics, Vol. 21, No. 4, 2000 , pp. 1-6.

[4] Wikipedia, "Laplace-Runge-Lenz Vector," 2013. http://en.wikipedia.org/wiki/Laplace $\% \mathrm{E} 2 \% 80 \% 93$ Runge $\% \mathrm{E} 2 \% 80 \% 93$ Lenz_vector

[5] W. R. Hamilton, "The Hodograph or a New Method of Expressing in Symbolic Language the Newtonian Law of Attraction," Proceedings of the Royal Irish Academy, Vol. 3, 1847, pp. 344-353.

[6] W. R. Hamilton, “Applications of Quaternions to Some Dynamical Questions," Proceedings of the Royal Irish Academy, Vol. 3, Appendix III, 1847, p. xxxvi-1.

[7] S. W. Groesberg, “Advanced Mechanics,” John Wiley \& Sons, Inc., Hopoken, 1998.

[8] H. Goldstein, C. P. Poole and J. L. Safko, "Classical Mechanics,” Addison Wesley, Boston, 2001.

[9] S. R. Spiegel, "Theoretical Mechanics," Schaum Outline Series, McGraw Hill Book Company, New York, 1967.

[10] W. Rindler, "Essential Relativity," Springr-Verlag, Berlin, 2006.

[11] F. D. Lawden, “Tensor Calculus and Relativity,” Chapman and Hall, London, 1975.

[12] L. D. Landau and E. M. Lifshitz, “The Classical Theory of Fields,” Pergamon International Library, Pergamon, 1980.

[13] http://en.wikipedia.org/wiki/Equivalence principle

[14] A. Einstein, "Relativity, the Special and General Theory," Henry Holt and Company, New York, 1920.

[15] C. Pollock. http://www.math.toronto.edu/ colliand/426_03/Papers03/C_Pollock.pdf

[16] L. P. Eisenhart, "Riemannian Geometry," Princeton University Press, Princeton, 1968. 